\title{
Diagnosis of chronic thromboembolic pulmonary hypertension
}

\author{
Deepa Gopalan ${ }^{1,2}$, Marion Delcroix ${ }^{3}$ and Matthias Held ${ }^{4}$
}

\begin{abstract}
Affiliations: ${ }^{1}$ Imperial College Hospitals, London, UK. ${ }^{2}$ Cambridge University Hospital, Cambridge, UK. ${ }^{3}$ University Hospitals of Leuven, Leuven, Belgium. ${ }^{4}$ Medical Mission Hospital, Dept of Internal Medicine, Center for Pulmonary Hypertension and Pulmonary Vascular Disease, Academic Teaching Hospital, JuliusMaximilian University of Würzburg, Würzburg, Germany.
\end{abstract}

$@$ ERSpublications

CTEPH is a curable form of pulmonary hypertension which requires multimodality imaging for accurate diagnosis http://ow.ly/oTVT308CcD4

Cite this article as: Gopalan D, Delcroix M, Held M. Diagnosis of chronic thromboembolic pulmonary hypertension. Eur Respir Rev 2017; 26: 160108 [https://doi.org/10.1183/16000617.0108-2016].

ABSTRACT Chronic thromboembolic pulmonary hypertension (CTEPH) is the only potentially curable form of pulmonary hypertension. Rapid and accurate diagnosis is pivotal for successful treatment. Clinical signs and symptoms can be nonspecific and risk factors such as history of venous thromboembolism may not always be present. Echocardiography is the recommended first diagnostic step. Cardiopulmonary exercise testing is a complementary tool that can help to identify patients with milder abnormalities and chronic thromboembolic disease, triggering the need for further investigation. Ventilation/perfusion $\left(V^{\prime} / Q^{\prime}\right)$ scintigraphy is the imaging methodology of choice to exclude CTEPH. Single photon emission computed tomography $V^{\prime} / Q^{\prime}$ is gaining popularity over planar imaging. Assessment of pulmonary haemodynamics by right heart catheterisation is mandatory, although there is increasing interest in noninvasive haemodynamic evaluation. Despite the status of digital subtraction angiography as the gold standard, techniques such as computed tomography (CT) and magnetic resonance imaging are increasingly used for characterising the pulmonary vasculature and assessment of operability. Promising new tools include dual-energy CT, combination of rotational angiography and cone beam CT, and positron emission tomography. These innovative procedures not only minimise misdiagnosis, but also provide additional vascular information relevant to treatment planning. Further research is needed to determine how these modalities will fit into the diagnostic algorithm for CTEPH.

\section{Introduction}

Chronic thromboembolic pulmonary hypertension (CTEPH) is the only form of pulmonary hypertension that is potentially curable. However, without appropriate treatment, CTEPH has an estimated 5-year survival of $30 \%$ in patients with mean pulmonary artery pressure (mPAP) $>40 \mathrm{mmHg}$ and $10 \%$ with mPAP $>50 \mathrm{mmHg}[1]$.

In recent times, there has been a resurgence of interest in the condition. This is the result of modification of existing treatments, such as pulmonary endarterectomy (PEA), to produce better clinical outcomes [2], the emergence of newer techniques such as balloon pulmonary angioplasty (BPA) and advances in pharmacotherapy. Swift and accurate diagnosis is a fundamental precondition for early establishment of relevant treatment. However, despite recent therapeutic progress, CTEPH remains underdiagnosed for a

This article has supplementary material available from err.ersjournals.com

Received: Nov 292016 | Accepted after revision: Jan 102017

Conflict of interest: Disclosures can be found alongside this article at err.ersjournals.com

Provenance: Publication of this peer-reviewed article was sponsored by Bayer AG, Berlin, Germany (principal sponsor, European Respiratory Review issue 143).

Copyright CERS 2017. ERR articles are open access and distributed under the terms of the Creative Commons Attribution Non-Commercial Licence 4.0. 
multitude of reasons, encompassing clinical barriers, an ill-informed imaging community, nonuniformity in diagnostics despite international guidelines and delay in referral to expert centres. This review summarises the diagnostic algorithm and nonimaging and functional examinations necessary for patients with CTEPH. It also focuses on the necessary imaging criteria and modalities required to formulate a diagnosis and determine extent and severity of disease, thereby assisting management decisions. Up-and-coming imaging developments are explored.

\section{The way into the diagnostic process: symptoms and clinical findings leading to the} suspicion of CTEPH

Although a definitive prediction of the true incidence of CTEPH after acute pulmonary embolism is difficult, due to variations in the nature and size of screened populations, imaging modalities and criteria used for diagnosis and follow-up duration, the estimated value ranges from $0.4 \%$ to $8.8 \%[3,4]$. While up to $25 \%$ of patients with CTEPH may have no history of previous pulmonary embolism [5], a substantial number of patients diagnosed with acute pulmonary embolism might already have underlying chronic disease with previously unidentified pulmonary hypertension $[6,7]$.

Estimated incidences of CTEPH, based on numbers of acute thromboembolic events and reported data derived from follow-up studies, are seemingly different to the actual number of diagnosed cases in expert centres [3-5]. This might be partly explained by lack of clinical awareness of CTEPH and inadequate technical knowledge of the various diagnostic modalities among community care physicians. Although tremendous progress has been made during recent years in developing advanced investigative tools and strategies, patients must still be referred for relevant tests based on clinical suspicion, with the important caveat that none of the noninvasive modalities have $100 \%$ sensitivity.

Some patients enter the diagnostic process with unclear symptoms suggestive of pulmonary hypertension, while others are suspected to have CTEPH based on their history, risk factors and clinical examination. Symptoms of CTEPH include exertional breathlessness, fatigue and angina $[5,8]$, with syncope and oedema reported in late stages of disease progression. These nonspecific symptoms can be potentially misleading, hence appropriate evaluation is often delayed and the correct diagnosis is made only after a protracted journey $[5,8,9]$. Presentation of comorbidities might also complicate the diagnosis. A high level of suspicion should be entertained in the evaluation of patients with risk factors such as recurrent venous thromboembolism, antiphospholipid antibodies and lupus anticoagulant, inflammatory bowel disease, ventriculoatrial shunt, cardiac pacemaker, a history of splenectomy or thyroid hormone replacement [10]. Patients who are diagnosed with acute pulmonary embolism but show signs of chronic disease [6] should be explored for CTEPH.

ECG signs of right heart strain include P-pulmonale, right bundle branch block, T-wave abnormalities in the chest leads and right-axis deviation [11-13]. Pulmonary vascular disease should be considered in patients with dyspnoea and normal flow and volumes but reduced transfer factor on lung function tests [14-16]. While decreased carbon dioxide levels are a recognised feature of idiopathic pulmonary arterial hypertension [17], hyperventilation and ineffective ventilation are even more pronounced in CTEPH [18]. Central sleep apnoea and Cheyne-Stokes respiration can be caused by CTEPH and should trigger further investigation [19].

\section{Echocardiography}

For patients with symptoms, signs and a history suggestive of pulmonary hypertension or CTEPH, or in those with dyspnoea of uncertain origin, the current guidelines recommend echocardiography as the first diagnostic step [11]. Evaluations include estimating peak velocity of tricuspid valve regurgitation, calculation of atrioventricular pressure gradients and detection of indirect signs of pulmonary hypertension such as right atrial and right ventricular dilatation, reduced right ventricular contractility and Doppler flow abnormalities in the right ventricular outflow tract [11]. However, these findings may be absent in the early stages of disease and echocardiography has been shown to miss pulmonary hypertension in as many as $10-31 \%$ of cases $[16,20,21]$.

\section{Cardiopulmonary exercise testing}

Hyperventilation in pulmonary arterial hypertension and CTEPH typically appears as ineffective ventilation [18-21]. Hence, cases of hyperventilation should be examined carefully to avoid being misclassified as having an idiopathic or psychogenic cause. In contrast to patients with psychogenic hyperventilation, patients with ineffective ventilation due to pulmonary vascular obstruction present with elevated alveolar-capillary gradients of oxygen and carbon dioxide [18-21]. This might be helpful to identify those with underlying pulmonary vascular disease (figure 1). In a retrospective study, cardiopulmonary exercise testing (CPET) was able to detect CTEPH despite normal echocardiography [21]. Although prospective evaluation of larger 

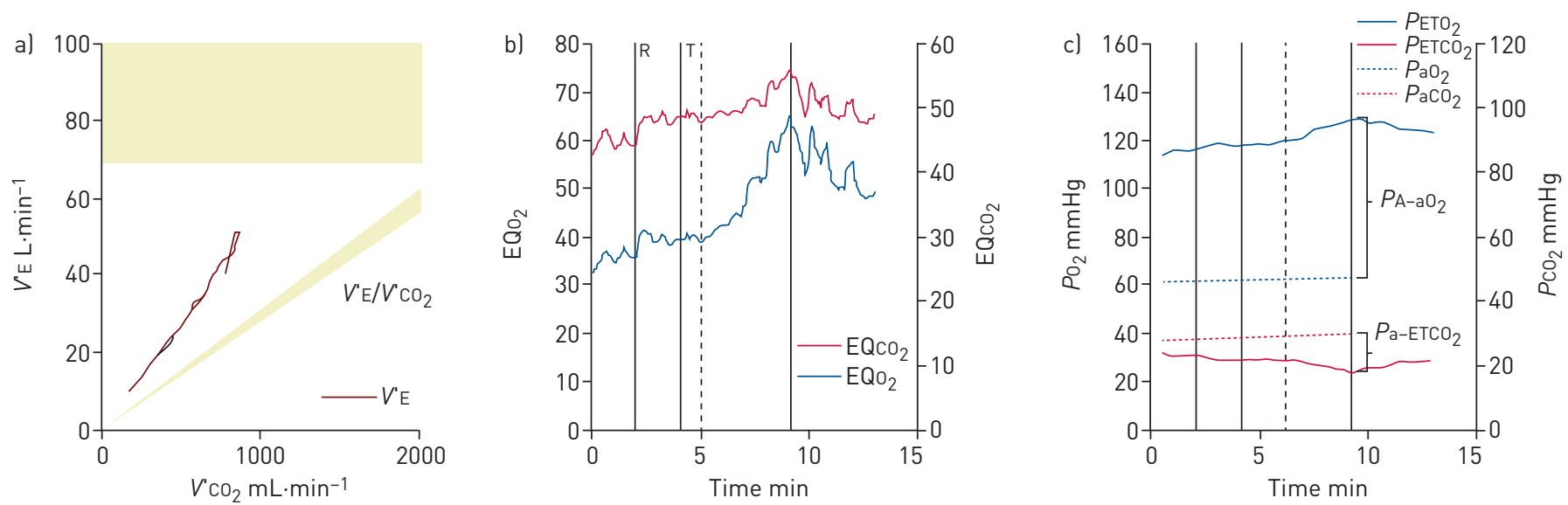

FIGURE 1 Cardiopulmonary exercise testing of a patient with chronic thromboembolic pulmonary hypertension showing fields 4,6 and 9 of the Wasserman panel. a) Elevated slope of minute ventilation $\left(V^{\prime} \mathrm{E}\right) /$ carbon dioxide output $\left(V^{\prime} \mathrm{CO}_{2}\right)$ ratio showing hyperventilation (field 4$)$; b) elevated ventilator equivalents for oxygen $\left(\mathrm{EQO}_{2}\right)$ and carbon dioxide $\left(\mathrm{EQCO}_{2}\right)$ showing ineffective ventilation (field 6$)$; $\mathrm{c}$ ) low and decreasing end-tidal carbon dioxide tension $\left(\mathrm{PETCO}_{2}\right)$, elevated alveolar-arterial oxygen tension gradient $\left(\mathrm{PA}_{\mathrm{A}-\mathrm{a}_{2}}\right)$ and elevated arterial-end-tidal carbon dioxide gradient $\left(P_{\mathrm{aa}} \mathrm{ETCO}_{2}\right)$ (field 9). $P \mathrm{ETO}_{2}$ : end-tidal oxygen tension; $\mathrm{PaO}_{2}$ : arterial oxygen tension; $P_{\mathrm{aCO}_{2}}$ : arterial carbon dioxide tension; $P \mathrm{O}_{2}$ : oxygen tension; $\mathrm{PCO}_{2}$ : carbon dioxide tension.

cohorts is still lacking, CPET is a promising complementary diagnostic tool for functional evaluation of patients with pulmonary vascular disease.

The entity of chronic thromboembolic disease (CTED) has been described and is characterised by chronic thromboembolic pulmonary vascular obstruction with normal resting pulmonary artery pressures [22]. Despite normal resting haemodynamics, this cohort of patients does show improvement after PEA [22]. Standard echocardiography will miss this diagnosis, but CPET can provide invaluable assistance in revealing similar findings in patients with CTED and CTEPH [23, 24].

When pulmonary hypertension is confirmed by the abovementioned investigations, it is imperative to exclude CTEPH unless there is sufficient evidence of left heart or pulmonary disease [11]. Imaging not only helps to detect chronic thromboembolism, but can also help with the choice of suitable therapy.

\section{Imaging}

\section{Ventilation/perfusion scintigraphy}

Ventilation/perfusion $\left(V^{\prime} / Q^{\prime}\right)$ scintigraphy is the imaging methodology of choice for exclusion of CTEPH. A normal $V^{\prime} / Q^{\prime}$ scan effectively excludes CTEPH with a sensitivity of $90-100 \%$ and a specificity of $94-100 \%$ [11]. The European Society of Cardiology guidelines on the diagnosis and management of acute pulmonary embolism advocate $V^{\prime} / Q^{\prime}$ as the first-line test for diagnosing CTEPH following acute pulmonary embolism [25]. However, in the wider clinical setting there is reduced utilisation of $V^{\prime} / Q^{\prime}$ scintigraphy leading to a diagnostic care gap, as some clinicians deem it to be irrelevant despite the standard recommendations [26].

It is important to remember that although the typical natural history of acute pulmonary embolism is complete or near complete resolution within 30 days [27], $>50 \%$ of patients can have persistent perfusion defects 6 months after the initial event, after which time thrombus resolution reaches a plateau phase [28]. The cardinal finding in pulmonary embolism is that of preserved ventilation and absent perfusion within a lung segment. However, it is well recognised that such a $V^{\prime} / Q^{\prime}$ mismatch can occur in a variety of conditions, including pulmonary artery sarcoma, vasculitis, venoocclusive disease, fibrosing mediastinitis and congenital pulmonary vascular abnormalities (figure 2). Although some centres only perform the perfusion component of the $V^{\prime} / Q^{\prime}$ scan, the ventilation data are often useful in defining the lung borders, aiding the detection of smaller peripheral perfusion defects. Furthermore, ventilation data provide additional information about alternative cardiopulmonary conditions, such as chronic obstructive lung disease, heart failure and pneumonia. Overall, the combination of ventilation and perfusion improves the specificity of the technique [29].

Single photon emission computed tomography (SPECT) $V^{\prime} / Q^{\prime}$ has increasingly gained popularity over planar imaging in the setting of acute pulmonary embolism due to its higher sensitivity and specificity, particularly in the presence of comorbidities [30, 31]. A well-known pitfall of planar $V^{\prime} / Q^{\prime}$ is "shine-though masking", which occurs due to the superposition of abnormal areas of perfusion over regions with normal perfusion, resulting in underestimation of the presence and extent of pulmonary embolism. Likewise, misinterpretation can happen where there is nonocclusive disease resulting in a rather underwhelming $V^{\prime} / Q^{\prime}$. These limitations can be overcome with the multiplanar capability of SPECT, as it can provide better defect 
a)

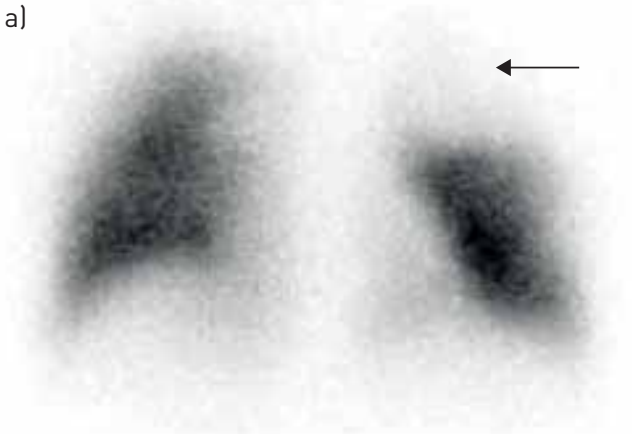

b)
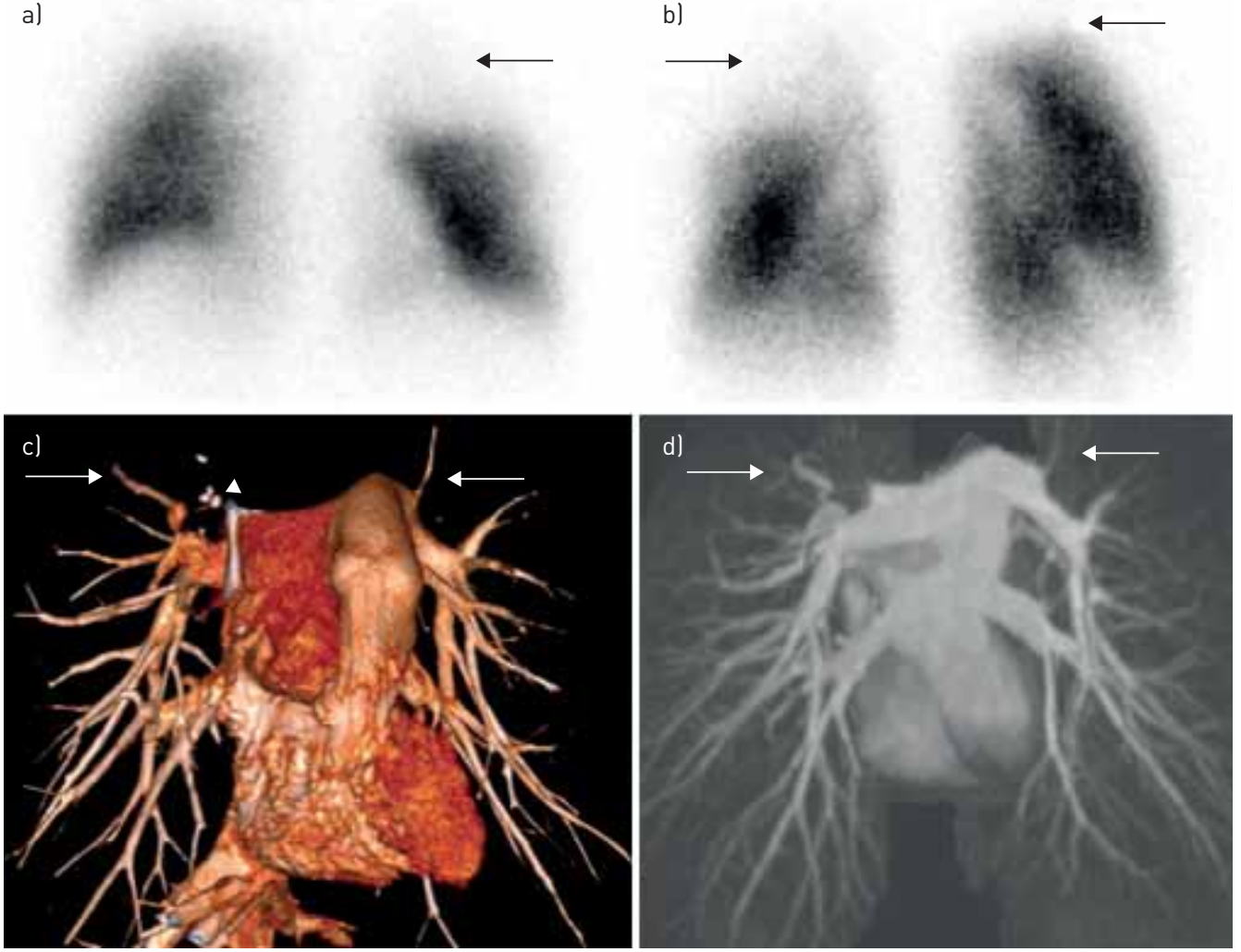

FIGURE 2 A 53-year-old patient with fibrosing mediastinitis. a) Anterior and b) posterior views from a perfusion scintigraphy scan show multiple segmental perfusion defects (arrows). c) Volume-rendered computed tomography angiography and d) magnetic resonance pulmonary angiography demonstrate attenuated pulmonary vasculature (arrows) with calcified mediastinal lymph nodes (arrowhead).

delineation with less interobserver variation, in addition to improved quantification [32]. While there is extensive literature on the diagnostic performance of planar $V^{\prime} / Q^{\prime}$, the data for $V^{\prime} / Q^{\prime}$ SPECT in CTEPH are sparse. Extrapolating the diagnostic performance of SPECT $V^{\prime} / Q^{\prime}$ in acute pulmonary embolism, it is likely that SPECT will supersede planar imaging over the next few years.

Irrespective of the technique, $V^{\prime} / Q^{\prime}$ reporting should follow holistic principles rather than antiquated rules. The European Association of Nuclear Medicine guidelines for $V^{\prime} / Q^{\prime}$ strongly encourage a move away from probabilistic scores and promote "Gestalt" interpretation, based on the integration of different sets of criteria and the observer's own experience [33]. In the context of CTEPH, there is a consensus that $V^{\prime} / Q^{\prime}$ readings should be binary, i.e. positive or negative. Although this may seem like an oversimplification in the setting of pulmonary hypertension, this approach can work, as a negative $V^{\prime} / Q^{\prime}$ result excludes group 4 conditions while a positive $V^{\prime} / Q^{\prime}$ requires downstream anatomical testing.

\section{Pulmonary vascular macro- and microcirculation}

The morphology of the diseased pulmonary arteries can be characterised noninvasively, using computed tomography (CT) or magnetic resonance (MR) angiography, or invasively, using traditional catheter pulmonary angiography. The CTEPH diagnostic criteria first described by AUGER et al. [34] in 1992 remain fundamentally similar to those currently used, albeit with a few modifications that have evolved with technological advances. The principal criteria are complete occlusion, pouch defects, intimal irregularity, intravascular webs and bands, stenosis and post-stenotic dilatation and abrupt tapering and truncation.

\section{CT pulmonary angiography}

Wide availability and ease of performance, in conjunction with robustly high sensitivity, specificity and accuracy, have made CT pulmonary angiography (CTPA) the imaging technique of choice for acute pulmonary embolism. In contrast, its role in CTEPH is less well defined.

Optimisation of acquisition and post-processing parameters are pivotal to avoid misdiagnosis. Short breath hold (3-5s) acquisition reduces respiratory motion artefacts. Thin collimation and thin-slice $(\leqslant 1 \mathrm{~mm})$ reconstruction in a soft-tissue kernel and analysis in the three-dimensional mode improve the display of 
subsegmental vessels as well as thin webs and bands. Generally, CTPA acquisition is not ECG gated, but there is evidence that ECG-gated images are superior to contrast-enhanced MR angiography and digital subtraction angiography for the depiction of the segmental and subsegmental arteries [35]. Retrospective gating (spiral mode) also allows for quantitative assessment of right ventricular function, with clearer demonstration of septal position and movement, but the radiation penalty precludes this from routine usage. To use the advantages of ECG gating, such as better vessel display, with sharp margins and avoidance of pulsation artefacts, prospective gating in the diastolic phase can be an acceptable compromise, as there will be marked reduction in the radiation dose, albeit with reduced capability of right ventricular function analysis.

The vascular abnormalities of CTEPH that can be seen on CTPA have been described extensively in the literature. Enlargement of central pulmonary arteries with decreased diameters of peripheral vessels is a rather generic feature. Eccentric wall-adherent thrombi, which may be calcified, are a more specific finding of CTEPH and are very different from the central filling defect within a distended lumen that is the hallmark of acute pulmonary embolism. Although not a specific feature, bronchial artery dilatation is frequently seen in CTEPH and has been shown to be associated with lower postoperative mortality and pulmonary vascular resistance (PVR) after PEA [36]. In addition, there is correlation between the total cross-sectional area of the bronchial arteries and the extent of central thromboembolic disease [37], as well as the severity of bronchopulmonary shunting on MR imaging [38]. It is important to recognise that a CT study optimised for the pulmonary circulation may miss the presence of bronchial circulation, as the latter is a branch of the descending thoracic aorta and ideally would require an examination that includes the systemic circulation.

Mosaic attenuation of the lung parenchyma resulting in geographical variation in perfusion characterised by sharply demarcated regions of hypo-attenuation is a frequent feature of CTEPH [39], and its presence can be a useful discriminator for chronicity in patients with suspected acute-on-chronic disease. Atelectatic bands, wedge-shaped infarcts, cavitating lesions and peripheral linear opacities are not specific, but are indicative of parenchymal scars. All of these findings require interrogation of the data in the appropriate "lung window" mode.

CTPA has high sensitivity and specificity in detecting thromboembolic disease at the lobar (97-100\% and 95-100\%, respectively) and segmental ( $86-100 \%$ and $93-99 \%$, respectively) levels [35, 40, 41]. It is important to note that while CTPA has been proven to be noninferior to $V^{\prime} / Q^{\prime}$ in diagnosing CTEPH [42], a negative CTPA does not exclude CTEPH, as subsegmental disease can be missed if reliance is placed only on CT.

Once the diagnosis of CTEPH is confirmed, CTPA can be used for assessment of operability. CT can provide a vascular roadmap for surgical planning and is the best modality for delineation of the proximal extent of the organised thromboembolic material [35], with good endarterectomy plane correlation. However, it must be emphasised that the use of CT for estimation of surgical suitability requires considerable imaging expertise and is usually best performed by high-volume, experienced institutions.

There is a large knowledge gap among the imaging community in the interpretation of CT in CTEPH, as the rarity of disease combined with the lack of expertise results in variable exposure to the condition among radiologists.

\section{Dual-energy CT}

Dual-energy CT (DECT) is a promising emerging tool that evaluates the material decomposition of iodine by simultaneous acquisition of two datasets at different tube voltages. The processed data generate conventional grey-scale images, colour-coded overlays highlighting the iodine distribution and virtual nonenhanced images from post-contrast images by using iodine-subtraction techniques. The resulting vascular perfusion pattern allows for qualitative assessment of hypoperfused lungs comparable to scintigraphy $[43,44]$. The calculation of iodine distribution in the lung parenchyma (pulmonary/perfused blood volume (PBV) maps) offers qualitative and quantitative insights into pulmonary haemodynamics [45]. The PBV score has been shown to correlate with mPAP and PVR measurements on right heart catheterisation, and hence can be used to estimate CTEPH severity [46], although there is no direct prospective evidence to suggest that PBV defects on the initial presentation predict CTEPH evolution.

In addition, a strong correlation exists between PBV imaging and mosaic attenuation [44]. Concordant PBV defects and hypo-attenuating areas of mosaic attenuation are indicative of a vascular cause, while discordance suggests small airways disease. The combination of DECT intravascular and parenchymal enhancement can also be used to determine whether hypoperfused regions have corresponding focal vascular obstruction. This will be of particular benefit in patients with distal CTEPH, where there is no overt intraluminal clot, but the diagnosis of CTEPH will not be missed due to the presence of typical perfusion defects (discrete, segmental and sharply defined) and mosaic attenuation. The dynamic combination of DECT for morphological vascular evaluation and exquisite PBV functional information is more advantageous compared to sequential $V^{\prime} / Q^{\prime}$ scintigraphy and traditional CTPA (figure 3). This concept is the subject of ongoing evaluation. 

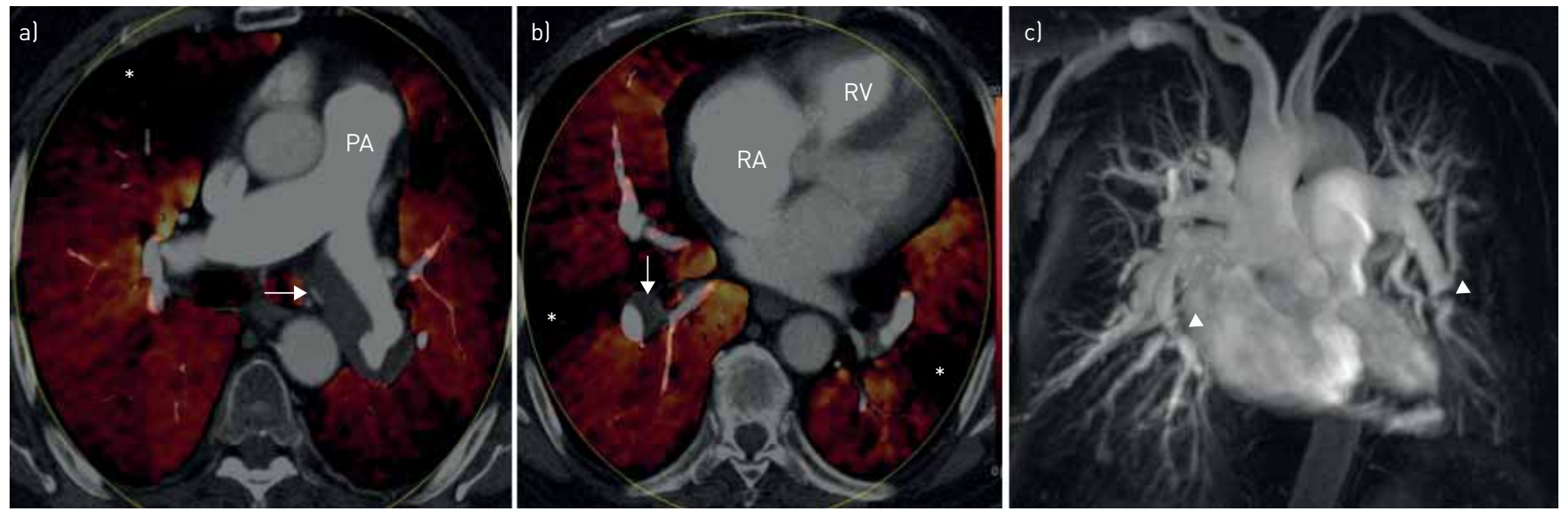

FIGURE 3 A 45-year-old with pulmonary hypertension. a, b) Dual-energy computed tomography pulmonary angiography and c) corresponding magnetic resonance pulmonary angiography confirm chronic thromboembolic pulmonary hypertension. There is dilatation of the main pulmonary artery (PA), dilated right atrium (RA) and right ventricle (RV) with right ventricular hypertrophy, eccentric thrombus in right lower lobe and left main pulmonary artery (arrows), multiple segmental perfusion defects (*), proximal pulmonary arterial stenosis and webs (arrowheads).

\begin{abstract}
Digital subtraction angiography
Catheter pulmonary angiography is still considered the gold standard for the assessment of pulmonary vasculature, but its routine use for diagnosis of CTEPH is being challenged by advances in noninvasive technology (figure 4). In a study of 24 patients, CT angiography (CTA) outperformed digital subtraction angiography (DSA) for detection of CTEPH [35]. The sensitivity of DSA ranged from $66 \%$ at main/lobar and $76 \%$ at segmental level, compared with $100 \%$ for CTA at comparable levels. DSA had a slight advantage over CTA at depicting subsegmental arteries (DSA 97\%; CTA 80\%), but the specificity of both techniques was excellent (100\%). One acknowledged reason for the relatively lower sensitivities of DSA in this study compared with previous work is that the technical quality of DSA was lower than that for noninvasive techniques. The authors rightly felt that this was a reflection of routine clinical practice, particularly in centres where the interventional skill of imaging specialists has been largely superseded by $\mathrm{CT}$, particularly in light of its relative ease of performance and accessibility. However, it must be emphasised that one supreme advantage of DSA is the ability to measure pulmonary arterial haemodynamics during right heart catheterisation. In addition, there has been a resurgence of interest in traditional angiography, due to the advent of BPA as a viable treatment option for selected patients with CTEPH who are unsuitable for surgery.
\end{abstract}
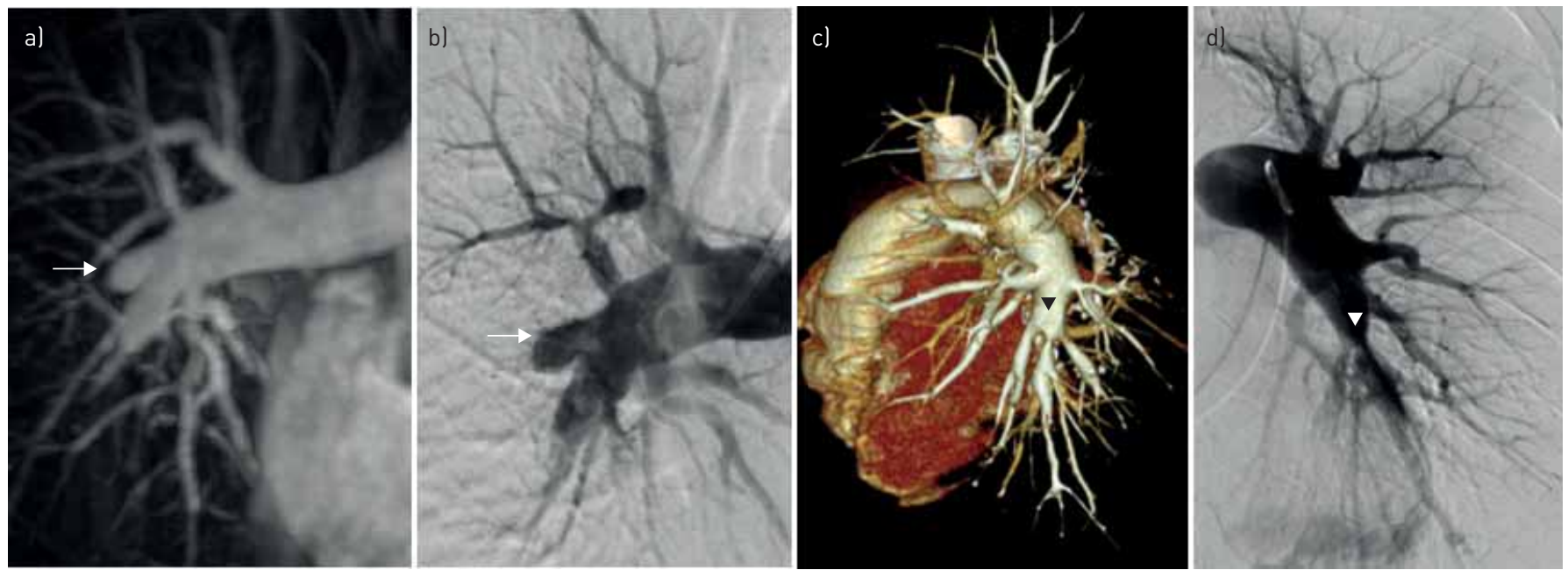

FIGURE 4 al Magnetic resonance and b) catheter pulmonary angiography and c) computed tomography and d) catheter pulmonary angiography in two different cases of chronic thromboembolic pulmonary hypertension. There is good disease correlation on all three modalities, e.g. pouch defect on magnetic resonance image (arrows) and proximal stenosis in left lower lobe artery (arrowhead). 

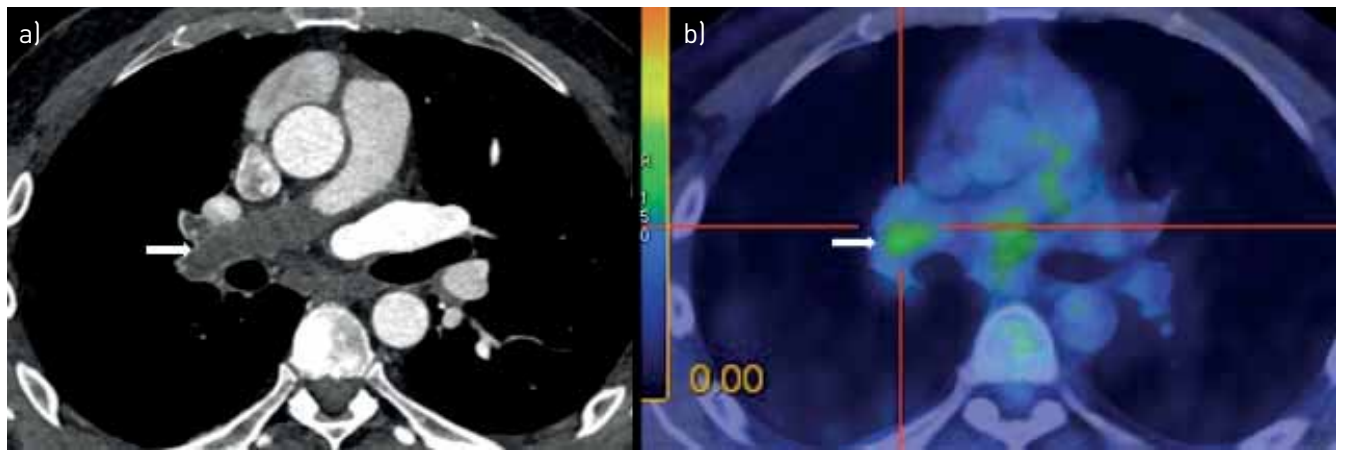

FIGURE 5 A 50-year-old male with persistent abnormality on computed tomography (CT) despite anticoagulation for 1 year. a) CT scan showing an expansile low attenuation lesion in the right main pulmonary artery (arrow); b) ${ }^{18} \mathrm{~F}$-fluorodeoxyglucose positron emission tomography with corresponding high uptake in keeping with pulmonary artery sarcoma. Note mediastinal soft tissue with increased uptake.

\section{Right heart catheterisation}

To confirm the diagnosis of pulmonary hypertension, and CTEPH in particular, right heart catheterisation is mandatory [11]. Measurement of pulmonary arterial occlusion pressure (PAOP) is necessary to exclude post-capillary pulmonary hypertension resulting from comorbidities. As in CTEPH, intravascular obstructions might confound the correct estimation of PAOP in some patients: left ventricular end-diastolic pressure in such patients should be obtained by left ventricular catheterisation. The guidelines recommend a complete haemodynamic evaluation by right heart catheterisation including cardiac output [11], because PVR is important to assess prognosis and the risks associated with PEA [47].

\section{Rotational angiography and cone beam CT}

This combination utilises flat-panel technology to provide intraprocedural images using either threedimensional rotational angiography (with contrast injection to visualise the blood vessels and high-contrast structures, such as bone and calcification) or cone-beam CT (without contrast injection to obtain CT-like soft-tissue contrast resolution). The CT images have high spatial resolution, but lower contrast resolution, increasing susceptibility to beam-hardening artefacts and noise. Image merging produces a three-dimensional roadmap that can be used in conjunction with real-time fluoroscopy, and provides the possibility of live monitoring from any angle and magnification. This can provide invaluable guidance for complex pulmonary vascular interventions [48-50].

\section{Cardiac MR imaging}

The place of MR imaging in the CTEPH management algorithm is very much dependent on local practice. Positive factors, such as wider availability and progressive technological improvement, with shorter acquisition times and larger volume coverage, have facilitated its growing acceptance.

With inherently high contrast resolution and spatial resolution, MR imaging has emerged as the reference standard in the assessment of right ventricular size and systolic function through the use of segmented balanced steady-state free precession cine sequences [51-53]. Cine imaging allows for morphological analysis of the right ventricle, which can be dilated and hypertrophied, with tricuspid regurgitation. Chronic right ventricle pressure overload leads to mechanical dyssynchrony due to flattening or convex bowing of the interventricular septum. This causes impairment of left ventricular diastolic filling with resultant reduction in left ventricular stroke volume and ejection fraction, and paradoxical movement of the interventricular septum. Semiquantitative analysis can provide an accurate and consistent estimation of biventricular functional parameters.

Contrast-enhanced MR angiography (ce-MRA) is the most effective MR technique for evaluation of the pulmonary macrocirculation. With acquisition times of $\sim 12-14 \mathrm{~s}$ for a high-resolution dataset, it is possible to get diagnostic images even in patients with dyspnoea. Evaluation of the source data and multiplanar reformations are pivotal to extract the maximum morphological information, while rotating maximum-intensity projections can furnish an overview of the pulmonary vasculature in its entirety (online supplementary figure S1 and video). MRA is superior to DSA in depicting the precise proximal beginning of the thromboembolic material, with good correlation with origin of the dissection plane during PEA [54].

In the study by Ley et al. [35] comparing CTA, ce-MRA and DSA, the sensitivity and specificity of MRA for diagnosing disease at main/lobar level were $83.1 \%$ and $98.6 \%$, respectively, and at the segmental level were $87.7 \%$ and $98.1 \%$, respectively. The subsegmental arteries were demonstrated only in $75 \%$ of cases, compared 
with $87 \%$ by DSA. By obtaining a delayed phase sequence, MRA can delineate the bronchial circulation, with the ability to then calculate the bronchopulmonary shunt fraction using phase-contrast MR imaging.

The evaluation of pulmonary microcirculation involves application of time-resolved MRA techniques that can produce data regarding the perfusion of the lung parenchyma and a supplementary low spatial resolution image of the pulmonary arteries. CTEPH perfusion defects are segmental compared with the patchy deficits seen in pulmonary arterial hypertension [55]. Absolute quantification of perfusion is feasible, but requires determination of the arterial input function and is not routinely performed in clinical practice. Due to its unique advantages of noninvasiveness, lack of exposure to ionising radiation, superior diagnostic accuracy and consistent reproducibility, MR can be used for serial follow-up of patients before and after surgical treatment of CTEPH, as it can detect changes in parameters reflecting cardiac remodelling and pulmonary clearance [56]. After successful PEA, there is a reduction in pulmonary arterial diameter with concomitant increase in pulmonary peak velocities. The postoperative reduction in mPAP correlates well with the increase in right ventricular ejection fraction [57].

The fast multispectral capability of MR imaging lends itself to functional imaging of the lungs using hyperpolarised noble gases such as helium-3 and xenon-129, with the potential to produce regional information on normal and abnormal lung ventilation. This evolving application holds enormous potential for enhancing sensitivity and contrast in pulmonary imaging.

\section{Positron emission tomography}

There is increasing interest in the utility of metabolic imaging in pulmonary hypertension. A recent study demonstrated increased uptake of glucose and fatty acids (using ${ }^{18} \mathrm{~F}$-fluorodeoxyglucose (FDG) and $\beta$-methyl-p- ${ }^{123}$ I-iodophenyl-pentadecanoic acid (BMIPP), respectively) in the right ventricle in patients with CTEPH with reduction in uptake post-PEA [58]. BMIPP accumulation in the right ventricle also correlates well with pulmonary haemodynamics [59]. Interesting though these findings are, the role of positron emission tomography (PET) in CTEPH is primarily to distinguish it from other conditions that can mimic the disease and so avoid misdiagnosis and minimise delay in the establishment of appropriate treatment (figure 5). Pulmonary artery sarcoma (PAS) exhibits a distinct difference in the specific uptake value (SUV max) on PET in comparison to CTEPH. In general, SUVmax of PAS is approximately three-fold or more compared with thrombi [60]. Using an SUVmax cut-off of 3.5, PET-CT has been shown to have sensitivity, specificity and accuracy of $100 \%$ in the diagnosis of malignant pulmonary artery lesions, with an average value of 10.2 (range 4.9-42.5) for PAS versus 1.7 (range 1.3-2.1) for thromboembolic disease [61]. Although thrombi in general do not bind FDG avidly, proximal vessels with thrombi show higher SUVmax compared with vessels without thrombi [62]. Pulmonary infarctions can exhibit high FDG uptake and be mistaken for satellite metastatic nodules in the lung periphery. This uptake is attributed to the composition of the thrombus (organised tissue and inflammatory cells) and is proportional to the numbers of macrophages or neutrophils, rather than the lesion size [63].

Other conditions that show high vascular FDG uptake are forms of vasculitis, such as Takayasu's arteritis, due to macrophage-rich areas within an area of inflammation. There is usually more pronounced uptake in the thoracic vessels, particularly at the level of the aortic arch and subclavian arteries, and this is prevalent in patients with predominantly systemic complaints [64]. It is important to remember that quiescent vasculitis may not show any avidity for FDG.

\section{Noninvasive assessment of pulmonary haemodynamics}

Attempts to correlate noninvasively measured haemodynamic parameters with right heart catheter data have met with limited success. The small number of comparable studies, paucity of prospective clinical cohorts, inevitable delays between noninvasive examinations and invasive right heart catheterisaton and the attendant haemodynamic variations that can happen even in a short time frame, are a few of the reasons that make data validation challenging.

Pulmonary artery distensibility derived from the relative cross-sectional area change between systole and diastole obtained by vessel area segmentation throughout the cardiac cycle is a reliable parameter for identifying pulmonary hypertension. A cut-off value of $16.5 \%$ was shown to have sensitivity and specificity of $86 \%$ and $96 \%$, respectively, for diagnosing pulmonary hypertension [65], but correlations with mPAP and PVR were moderate $[66,67]$.

Interventricular septal (IVS) curvature may predict mortality and clinical outcome in patients with CTEPH. The MR-derived measurement of IVS curvature is proportional to systolic (s)PAP, with a value $>67 \mathrm{mmHg}$ observed when leftward curvature is present [68]. This finding was extended to include mPAP as well as sPAP and further validated using ECG-gated 320-slice CT imaging [41]. On the basis of multiple linear regression analysis, $\mathrm{mPAP}$ has been shown to be computable exclusively from MR phase-contrast flow measurements [69]. Good inverse correlation has been demonstrated between MPAP and sPAP and 
MR-derived pressure-wave velocity [66], and DECT-derived PBV values also correlate with pulmonary haemodynamics [70].

The Cobb angle is defined as the angle between the interventricular septum and the line between the sternal midpoint and thoracic vertebral spinous process, and is measured during diastole. CT-based measurement of Cobb angle correlates with PVR and $N$-terminal prohormone of brain natriuretic peptide level. A cut-off value of $67.55^{\circ}$ has $72.5 \%$ sensitivity and $84 \%$ specificity for predicting PVR $\geqslant 1000$ dyn $\cdot \mathrm{s} \cdot \mathrm{cm}^{-5}$ [71]. In addition, MR models to estimate PVR obtained using a derivation cohort based on physiological plausibility and statistical criteria have been successful in demonstrating the feasibility of purely MR-based detection of increased PVR with high accuracy [72, 73]. The mean average pulmonary artery velocity had the strongest univariate correlation with PVR, irrespective of the cause and severity of pulmonary hypertension [72].

An MR study [74] was able to duplicate the earlier echocardiographic finding of an abnormal mid-systolic velocity deceleration (notch) in patients with CTEPH [75] and demonstrated its disappearance following PEA in parallel with an increase of peak velocity.

Multidirectional phase-contrast MR imaging is a propitious tool for assessment of pulmonary haemodynamics [76, 77]. Although there is no published literature about its potential use in CTEPH, its unique ability to provide vectorial flow data promises to give new insights into pulmonary arterial physiology. Vortical blood flow in the main pulmonary artery develops when $\mathrm{mPAP}$ exceeds $16.0 \mathrm{mmHg}$, and its duration increases linearly with mPAP. A vortical flow that is $>14.3 \%$ of the cardiac interval corresponds to pulmonary hypertension with a sensitivity of $97 \%$ and specificity of $96 \%$ [78, 79]. A publication using multivariate modelling that included relative area change of the main pulmonary artery, and flow characteristics also demonstrated the feasibility of estimating PVR in pulmonary hypertension [80]. Four-dimensional flow could be explored to identify patients who are at risk for developing pulmonary hypertension following acute pulmonary embolism or serial monitoring of patients who have CTED as opposed to CTEPH to work out ideal timing of surgery.

\section{Conclusion}

Routine screening for CTEPH after pulmonary embolism is not supported by current evidence. However, in symptomatic patients with typical risk factors and clinical findings suggestive of chronic thromboembolic vascular disease, careful clinical evaluation and imaging is necessary to confirm or exclude CTEPH. Current guidelines recommend that all patients with suspected CTEPH be referred to an expert centre for accurate differential diagnosis and operability assessment to be carried out by a multidisciplinary team. Noninvasive imaging techniques are complementary and not competing modalities in the investigation and management of CTEPH. Currently, $V^{\prime} / Q^{\prime}$ scintigraphy remains the screening method of choice. Right heart catheterisation is mandatory for haemodynamic evaluation. While a combination of CTPA for morphological delineation and MR imaging for functional and pulmonary vascular assessment with catheter angiography reserved as a problem-solving tool is a safe approach, the authors acknowledge that there might be difficulties in didactic implementation of such a proposition due to lack of appropriate equipment and/or expertise. Given that MR imaging is an expensive examination with limited availability, selective pulmonary angiography is likely to be required in most patients to identify those who may benefit from surgery or angioplasty.

Newer developments, such as dual-energy iodine mapping that can act as a surrogate marker of lung perfusion may in the future replace $V^{\prime} / Q^{\prime}$ scintigraphy, but it must be emphasised that in the current global financial climate, the cheaper and reliable $V^{\prime} / Q^{\prime}$ is unlikely to be ousted by more expensive technologies in the near future or in a more widespread fashion. The feasibility of noninvasive assessment of haemodynamic parameters has been established, but further validation with reproducibility studies in large patient populations is clearly warranted. The use of four-dimensional flow techniques to detect latent pulmonary hypertension is intriguing, but this is still an area of active research and requires standardisation prior to integration into routine clinical use.

\section{Acknowledgements}

Editorial assistance was provided by Adelphi Communications Ltd (Bollington, UK), supported by Bayer AG (Berlin, Germany).

\section{References}

1 Fedullo P, Kerr KM, Kim NH, et al. Chronic thromboembolic pulmonary hypertension. Am J Respir Crit Care Med 2011; 183: 1605-1613.

2 Jenkins D, Madani M, Fadel E, et al. Pulmonary endarterectomy in the management of chronic thromboembolic pulmonary hypertension. Eur Respir Rev 2017; 26: 160111. 
3 Dentali F, Donadini M, Gianni M, et al. Incidence of chronic pulmonary hypertension in patients with previous pulmonary embolism. Thromb Res 2009; 124: 256-258.

4 Poli D, Grifoni E, Antonucci E, et al. Incidence of recurrent venous thromboembolism and of chronic thromboembolic pulmonary hypertension in patients after a first episode of pulmonary embolism. J Thromb Thrombolysis 2010; 30: 294-299.

5 Pepke-Zaba J, Delcroix M, Lang I, et al. Chronic thromboembolic pulmonary hypertension (CTEPH): results from an international prospective registry. Circulation 2011; 124: 1973-1981.

6 Guérin L, Couturaud F, Parent F, et al. Prevalence of chronic thromboembolic pulmonary hypertension after acute pulmonary embolism. Prevalence of CTEPH after pulmonary embolism. Thromb Haemost 2014; 112: 598-605.

7 Simonneau G, Torbicki A, Dorfmüller P, et al. The pathophysiology of chronic thromboembolic pulmonary hypertension. Eur Respir Rev 2017; 26: 160112.

8 Held M, Grün M, Holl R, et al. Chronisch thromboembolische pulmonale Hypertonie: Latenz bis zur Diagnosesicherung und klinischer Zustand bei Diagnosestellung. [Chronic thromboembolic pulmonary hypertension: time delay from onset of symptoms to diagnosis and clinical condition at diagnosis]. Dtsch Med Wochenschr 2014; 139: 1647-1652.

9 Mayer E, Jenkins D, Lindner J, et al. Surgical management and outcome of patients with chronic thromboembolic pulmonary hypertension: results from an international prospective registry. J Thorac Cardiovasc Surg 2011; 141: $702-710$.

10 Bonderman D, Wilkens H, Wakounig S, et al. Risk factors for chronic thromboembolic pulmonary hypertension. Eur Respir J 2009; 33: 325-331.

11 Galiè N, Humbert M, Vachiery JL, et al. 2015 ESC/ERS Guidelines for the diagnosis and treatment of pulmonary hypertension. The Joint Task Force for the Diagnosis and Treatment of Pulmonary Hypertension of the European Society of Cardiology (ESC) and the European Respiratory Society (ERS). Eur Respir J 2015; 46: 903-975.

12 Al-Naamani K, Hijal T, Nguyen V, et al. Predictive values of the electrocardiogram in diagnosing pulmonary hypertension. Int J Cardiol 2008; 127: 214-218.

13 Bonderman D, Wexberg P, Martischnig AM, et al. A noninvasive algorithm to exclude pre-capillary pulmonary hypertension. Eur Respir J 2011; 37: 1096-1103.

14 Sun XG, Hansen JE, Oudiz RJ, et al. Pulmonary function in primary pulmonary hypertension. J Am Coll Cardiol 2003; 41: 1028-1035.

15 Trip P, Nossent EJ, de Man FS, et al. Severely reduced diffusion capacity in idiopathic pulmonary arterial hypertension: patient characteristics and treatment responses. Eur Respir J 2013; 42: 1575-1585.

16 Coghlan JG, Denton CP, Grünig E, et al. Evidence-based detection of pulmonary arterial hypertension in systemic sclerosis: the DETECT study. Ann Rheum Dis 2014; 73: 1340-1349.

17 Hoeper MM, Pletz MW, Golpon H, et al. Prognostic value of blood gas analyses in patients with idiopathic pulmonary arterial hypertension. Eur Respir J 2007; 29: 944-950.

18 Scheidl SJ, Englisch C, Kovacs G, et al. Diagnosis of CTEPH versus IPAH using capillary to end-tidal carbon dioxide gradients. Eur Respir J 2012; 39: 119-124.

19 Held M, Meintz S, Baron S, et al. Surgical cure of central sleep apnea? Am J Respir Crit Care Med 2013; 188: 395-396.

20 Held M, Linke M, Jany B. Echokardiographie und Rechtsherzkatheterisierung bei pulmonaler Hypertonie. [Echocardiography and right heart catheterization in pulmonal hypertension]. Dtsch Med Wochenschr 2014; 139: 1511-1517.

21 Held M, Grün M, Holl R, et al. Cardiopulmonary exercise testing to detect chronic thromboembolic pulmonary hypertension in patients with normal echocardiography. Respiration 2014; 87: 379-387.

22 Taboada D, Pepke-Zaba J, Jenkins DP, et al. Outcome of pulmonary endarterectomy in symptomatic chronic thromboembolic disease. Eur Respir J 2014; 44: 1635-1645.

23 Held M, Kolb P, Grün M, et al. Functional characterization of patients with chronic thromboembolic disease. Respiration 2016; 91: 503-509.

24 McCabe C, Deboeck G, Harvey I, et al. Inefficient exercise gas exchange identifies pulmonary hypertension in chronic thromboembolic obstruction following pulmonary embolism. Thromb Res 2013; 132: 659-665.

25 Konstantinides SV, Torbicki A, Agnelli G, et al. 2014 ESC guidelines on the diagnosis and management of acute pulmonary embolism. Eur Heart J 2014; 35: 3033-3069.

26 McLaughlin VV, Langer A, Tan M, et al. Contemporary trends in the diagnosis and management of pulmonary arterial hypertension: an initiative to close the care gap. Chest 2013; 143: 324-332.

27 Lang IM. Chronic thromboembolic pulmonary hypertension - not so rare after all. N Engl J Med 2004; 350: 2236-2238.

28 Nijkeuter M, Hovens MM, Davidson BL, et al. Resolution of thromboemboli in patients with acute pulmonary embolism: a systematic review. Chest 2006; 129: 192-197.

29 Creutzig H, Gonda S, Creutzig A, et al. Frequencies of segmental perfusion and ventilation abnormalities in lung scintigraphy. Eur J Nucl Med 1983; 8: 401-403.

30 Gutte H, Mortensen J, Jensen CV, et al. Comparison of V/Q SPECT and planar V/Q lung scintigraphy in diagnosing acute pulmonary embolism. Nucl Med Commun 2010; 31: 82-86.

31 Quirce R, Ibáñez-Bravo S, Jiménez-Bonilla J, et al. Contribution of V/Q SPECT to planar scintigraphy in the diagnosis of pulmonary embolism. Rev Esp Med Nucl Imagen Mol 2014; 33: 153-158.

32 Bajc M, Olsson CG, Olsson B, et al. Diagnostic evaluation of planar and tomographic ventilation/perfusion lung images in patients with suspected pulmonary emboli. Clin Physiol Funct Imaging 2004; 24: 249-256.

33 Bajc M, Neilly JB, Miniati M, et al. EANM guidelines for ventilation/perfusion scintigraphy: part 1. Pulmonary imaging with ventilation/perfusion single photon emission tomography. Eur J Nucl Med Mol Imaging 2009; 36: 1356-1370.

34 Auger WR, Fedullo PF, Moser KM, et al. Chronic major-vessel thromboembolic pulmonary artery obstruction: appearance at angiography. Radiology 1992; 182: 393-398.

35 Ley S, Ley-Zaporozhan J, Pitton MB, et al. Diagnostic performance of state-of-the-art imaging techniques for morphological assessment of vascular abnormalities in patients with chronic thromboembolic pulmonary hypertension (CTEPH). Eur Radiol 2012; 22: 607-616. 
39 Schwickert H, Schweden F, Schild H, et al. Darstellung der chronisch rezidivierenden Lungenembolie mit der Spiral-CT. [Detection of chronic recurrent pulmonary emboli using spiral-CT]. RöFo 1993; 158: 308-313.

40 Reichelt A, Hoeper MM, Galanski M, et al. Chronic thromboembolic pulmonary hypertension: evaluation with 64-detector row CT versus digital substraction angiography. Eur J Radiol 2009; 71: 49-54.

41 Sugiura T, Tanabe N, Matsuura Y, et al. Role of 320-slice CT imaging in the diagnostic workup of patients with chronic thromboembolic pulmonary hypertension. Chest 2013; 143: 1070-1077.

42 He J, Fang W, Lv B, et al. Diagnosis of chronic thromboembolic pulmonary hypertension: comparison of ventilation/perfusion scanning and multidetector computed tomography pulmonary angiography with pulmonary angiography. Nucl Med Commun 2012; 33: 459-463.

43 Dournes G, Verdier D, Montaudon M, et al. Dual-energy CT perfusion and angiography in chronic thromboembolic pulmonary hypertension: diagnostic accuracy and concordance with radionuclide scintigraphy. Eur Radiol 2014; 24: 42-51.

44 Renard B, Remy-Jardin M, Santangelo T, et al. Dual-energy CT angiography of chronic thromboembolic disease: can it help recognize links between the severity of pulmonary arterial obstruction and perfusion defects? Eur $J$ Radiol 2011; 79: 467-472.

45 Nakazawa T, Watanabe Y, Hori Y, et al. Lung perfused blood volume images with dual-energy computed tomography for chronic thromboembolic pulmonary hypertension: correlation to scintigraphy with single-photon emission computed tomography. J Comput Assist Tomogr 2011; 35: 590-595.

46 Takagi H, Ota H, Sugimura K, et al. Dual-energy CT to estimate clinical severity of chronic thromboembolic pulmonary hypertension: comparison with invasive right heart catheterization. Eur J Radiol 2016; 85: 1574-1580.

47 Jenkins D, Mayer E, Screaton N, et al. State-of-the-art chronic thromboembolic pulmonary hypertension diagnosis and management. Eur Respir Rev 2012; 21: 32-39.

48 Fukuda T, Ogo T, Nakanishi N, et al. Evaluation of organized thrombus in distal pulmonary arteries in patients with chronic thromboembolic pulmonary hypertension using cone-beam computed tomography. Jpn J Radiol 2016; 34: 423-431.

49 Sugiyama M, Fukuda T, Sanda Y, et al. Organized thrombus in pulmonary arteries in patients with chronic thromboembolic pulmonary hypertension; imaging with cone beam computed tomography. Jpn J Radiol 2014; 32: 375-382.

50 Yanagisawa R, Kataoka M, Inami T, et al. Efficacy of 360-degree three-dimensional rotational pulmonary angiography to guide percutaneous transluminal pulmonary angioplasty. EuroIntervention 2014; 9: 1483.

51 Beygui F, Furber A, Delépine S, et al. Routine breath-hold gradient echo MRI-derived right ventricular mass, volumes and function: accuracy, reproducibility and coherence study. Int J Cardiovasc Imaging 2004; 20: 509-516.

52 Winter MM, Bernink FJ, Groenink M, et al. Evaluating the systemic right ventricle by CMR: the importance of consistent and reproducible delineation of the cavity. J Cardiovasc Magn Reson 2008; 10: 40.

53 Grothues F, Moon JC, Bellenger NG, et al. Interstudy reproducibility of right ventricular volumes, function, and mass with cardiovascular magnetic resonance. Am Heart J 2004; 147: 218-223.

54 Kreitner KF, Kunz RP, Ley S, et al. Chronic thromboembolic pulmonary hypertension - assessment by magnetic resonance imaging. Eur Radiol 2007; 17: 11-21.

55 Ley S, Fink C, Zaporozhan J, et al. Value of high spatial and high temporal resolution magnetic resonance angiography for differentiation between idiopathic and thromboembolic pulmonary hypertension: initial results. Eur Radiol 2005; 15: 2256-2263.

56 Berman M, Gopalan D, Sharples L, et al. Right ventricular reverse remodeling after pulmonary endarterectomy: magnetic resonance imaging and clinical and right heart catheterization assessment. Pulm Circ 2014; 4: 36-44.

57 Kreitner KF, Ley S, Kauczor HU, et al. Chronic thromboembolic pulmonary hypertension: pre- and postoperative assessment with breath-hold MR imaging techniques. Radiology 2004; 232: 535-543.

58 Sakao S, Daimon M, Voelkel NF, et al. Right ventricular sugars and fats in chronic thromboembolic pulmonary hypertension. Int J Cardiol 2016; 219: 143-149.

59 Sakao S, Miyauchi H, Voelkel NF, et al. Increased right ventricular fatty acid accumulation in chronic thromboembolic pulmonary hypertension. Ann Am Thorac Soc 2015; 12: 1465-1472.

60 Ito K, Kubota K, Morooka M, et al. Diagnostic usefulness of ${ }^{18}$ F-FDG PET/CT in the differentiation of pulmonary artery sarcoma and pulmonary embolism. Ann Nucl Med 2009; 23: 671-676.

61 Lee EJ, Moon SH, Choi JY, et al. Usefulness of fluorodeoxyglucose positron emission tomography in malignancy of pulmonary artery mimicking pulmonary embolism. ANZ J Surg 2013; 83: 342-347.

62 Wittram C, Scott JA. ${ }^{18}$ F-FDG PET of pulmonary embolism. AJR Am J Roentgenol 2007; 189: $171-176$.

63 Kamel EM, McKee TA, Calcagni ML, et al. Occult lung infarction may induce false interpretation of ${ }^{18}$ F-FDG PET in primary staging of pulmonary malignancies. Eur J Nucl Med Mol Imaging 2005; 32: 641-646.

64 Belhocine T, Blockmans D, Hustinx R, et al. Imaging of large vessel vasculitis with ${ }^{18}$ FDG PET: illusion or reality? A critical review of the literature data. Eur J Nucl Med Mol Imaging 2003; 30: 1305-1313.

65 Revel MP, Faivre JB, Remy-Jardin M, et al. Pulmonary hypertension: ECG-gated 64-section CT angiographic evaluation of new functional parameters as diagnostic criteria. Radiology 2009; 250: 558-566.

66 Sanz J, Kuschnir P, Rius T, et al. Pulmonary arterial hypertension: noninvasive detection with phase-contrast MR imaging. Radiology 2007; 243: 70-79.

67 Swift AJ, Rajaram S, Condliffe R, et al. Diagnostic accuracy of cardiovascular magnetic resonance imaging of right ventricular morphology and function in the assessment of suspected pulmonary hypertension results from the ASPIRE registry. J Cardiovasc Magn Reson 2012; 14: 40.

68 Roeleveld RJ, Marcus JT, Faes TJ, et al. Interventricular septal configuration at MR imaging and pulmonary arterial pressure in pulmonary hypertension. Radiology 2005; 234: 710-717. 
69 Kreitner KF, Wirth GM, Krummenauer F, et al. Noninvasive assessment of pulmonary hemodynamics in patients with chronic thromboembolic pulmonary hypertension by high temporal resolution phase-contrast MRI: correlation with simultaneous invasive pressure recordings. Circ Cardiovasc Imaging 2013; 6: 722-729.

70 Meinel FG, Graef A, Thierfelder KM, et al. Automated quantification of pulmonary perfused blood volume by dual-energy CTPA in chronic thromboembolic pulmonary hypertension. RöFo 2014; 186: 151-156.

71 Liu M, Ma Z, Guo X, et al. Cardiovascular parameters of computed tomographic pulmonary angiography to assess pulmonary vascular resistance in patients with chronic thromboembolic pulmonary hypertension. Int $J$ Cardiol 2013; 164: 295-300.

72 García-Alvarez A, Fernández-Friera L, Mirelis JG, et al. Non-invasive estimation of pulmonary vascular resistance with cardiac magnetic resonance. Eur Heart J 2011; 32: 2438-2445.

73 Swift AJ, Rajaram S, Hurdman J, et al. Noninvasive estimation of PA pressure, flow, and resistance with CMR imaging: derivation and prospective validation study from the ASPIRE registry. JACC Cardiovasc Imaging 2013; 6: 1036-1047.

74 Rolf A, Rixe J, Kim WK, et al. Pulmonary vascular remodeling before and after pulmonary endarterectomy in patients with chronic thromboembolic pulmonary hypertension: a cardiac magnetic resonance study. Int $J$ Cardiovasc Imaging 2015; 31: 613-619.

75 Hardziyenka M, Reesink HJ, Bouma BJ, et al. A novel echocardiographic predictor of in-hospital mortality and mid-term haemodynamic improvement after pulmonary endarterectomy for chronic thrombo-embolic pulmonary hypertension. Eur Heart J 2007; 28: 842-849.

76 Unterhinninghofen R, Ley S, Ley-Zaporozhan J, et al. Concepts for visualization of multidirectional phase-contrast MRI of the heart and large thoracic vessels. Acad Radiol 2008; 15: 361-369.

77 Reiter G, Reiter U, Kovacs G, et al. Magnetic resonance-derived 3-dimensional blood flow patterns in the main pulmonary artery as a marker of pulmonary hypertension and a measure of elevated mean pulmonary arterial pressure. Circ Cardiovasc Imaging 2008; 1: 23-30.

78 Reiter G, Reiter U, Kovacs G, et al. Blood flow vortices along the main pulmonary artery measured with MR imaging for diagnosis of pulmonary hypertension. Radiology 2015; 275: 71-79.

79 Reiter U, Reiter G, Kovacs G, et al. Evaluation of elevated mean pulmonary arterial pressure based on magnetic resonance 4D velocity mapping: comparison of visualization techniques. PLoS One 2013; 8: e82212.

80 Kheyfets VO, Schafer M, Podgorski CA, et al. 4D magnetic resonance flow imaging for estimating pulmonary vascular resistance in pulmonary hypertension. J Magn Reson Imaging 2016; 44: 914-922. 